\title{
Could dysbiosis of inflammatory and anti-inflammatory gut bacteria have an implications in the development of type 2 diabetes? A pilot investigation
}

\author{
Prasanna Kulkarni ${ }^{1}$, Poornima Devkumar ${ }^{1}$ and Indranil Chattopadhyay ${ }^{2^{*}}$ (])
}

\begin{abstract}
Objective: Differential alterations in gut microbiota and chronic low-grade inflammation play a critical role in the development of Type 2 diabetes (T2D). Here we aimed to investigate if dysbiosis of inflammation and anti-inflammation-associated gut bacterial communities in fecal samples of individuals had any influence on T2D using a 16S rRNA gene of $\mathrm{V} 3$ region sequencing at Illumina MiSeq platform.
\end{abstract}

Results: Our findings showed that a higher abundance of inflammatory bacteria such as Lactobacillus ruminis, Ruminococcus gnavus, Bacteroides caccae, Butyricimonas, and Collinsella aerofaciens, and lower abundance of anti-inflammatory bacteria such as Faecalibacterium prausnitzii, and Butyrivibrio that likely play a role in the development of T2D. Our findings hint the potential of indigenous microbiota in developing diagnostic markers and therapeutic targets in T2D.

Keywords: Gut microbiota, Type 2 diabetes, Inflammation, Diagnostic marker

\section{Introduction}

Type 2 diabetes (T2D) is a chronic metabolic disorder characterized by hyperglycemia, insulin resistance, and insufficient secretion of insulin [1]. Studies suggest that nature of gut microbiome determine the onset of T2D through alterations in glucose metabolism and abnormal production of short-chain fatty acids (SCFAs) such acetic acid, propionic acid, and butyric acid [2-4], which appears to hold relevance for the presence or absence of indigenous gut microbiota that appears to correlate with the levels of SCFAs in the host's gut compartment. The differential levels of gut bacteria holds key to determine the natural history and progression of several human diseases, for instance, tuberculosis, HIV infection, cancer

\footnotetext{
*Correspondence: indranil_ch@yahoo.com

${ }^{2}$ Department of Life Sciences, Central University of Tamil Nadu,

Thiruvarur 610005, India

Full list of author information is available at the end of the article
}

and several autoimmune, hypersensitivity and inflammatory manifestations. Accumulating lines of evidence suggests that lower abundance of butyrate producing bacteria such as Bifidobacterium, Roseburia, Akkermansia, Blautia, and Faecalibacterium prauznitzii contribute to the outcome of T2D [5-7]. It has also been reported that butyrate induces low-grade inflammation that drives the development of T2DM [8]. Genus Lactobacillus is responsible for chronic inflammation in T2D patients [9]. In this study, we hypothesized that dysbiosis of gut bacteria associated with inflammation and anti-inflammatory may have a role in the development of T2D. Significant alterations in the composition of gut microbiota may be considered as potential biomarkers to predict the existence or onset of T2D in individuals. Here, we aimed to examine the dysbiosis of gut microbial communities associated with inflammation in fecal samples of patients with T2D as well as healthy controls. 


\section{Main text}

\section{Materials and methods}

Fecal samples were collected from 5 patients with T2D (aged $56 \pm 8$ years) who were referred to the Institute of Ayurveda and Integrative Medicine (I-AIM), Bangalore, India during July 2017 to December 2017. The inclusion criteria of the current study were a diagnosis of T2D and hemoglobin A1c (HbA1c > 6.5\%). The exclusion criteria were as follows: (i) Type 1 and other specific types of diabetes (ii) T2D patients administering oral hypoglycemic drugs /insulin (iii) women who are pregnant, attempting to conceive, or nursing mothers, and/or participants on prolonged therapy (including antibiotics during the last 3 months) (iv) participants with one or more of the following known co-morbid conditions like retinopathy, neuropathy, and nephropathy, participants with acute and chronic gastrointestinal disorders like inflammatory bowel syndrome (IBS), food allergy and lactose intolerance (v) participants with a history of organ transplantation and diseases of the oral cavity. Stool specimens were also collected from 5 healthy individuals without diabetes and with no apparent history of IBS or any GI related problems that warrant long-term medications at least 6 months prior to recruitment in the study, matched for age, gender, and living environment. Healthy individuals recruited in the study had a BMI between 18.5 and 25, and did not have a history of antibiotic usage at least 3 months before participation in the investigation.

Fecal samples were collected in a commercial OMNIgene GUT tube (DNA Genotek) as per the manufacturer's instructions. DNA was extracted from stool samples by using Qiagen DNeasy blood and tissue kit as per manufacturer's guidelines. Ten nanograms of the genomic DNA was subjected to amplification, and sequencing of the $16 \mathrm{~S}$ rRNA gene using V3 regionspecific primers as per Illumina-recommended primer was performed on an Illumina MiSeq Sequencer (Illumina, Inc.). The Illumina paired end reads (150*2) were demultiplexed using the bcl2fastq tool. The paired end reads were quality checked using FastQC. The raw reads having primer sequence and high quality bases were selected, and the reads were further stitched using Fastq-join. These stitched reads were considered for further analysis using QIIME pipeline. The query sequences were clustered using UCLUST method. The taxonomy of these clusters was assigned based on $>=97 \%$ sequence similarity against the curated chimera free16s rRNA database (Greengenes $v$ 13.8). The functional analysis was done by using Phylogenetic Investigation of Communities by Reconstruction of Unobserved States (PICRUSt) tool.

\section{Results}

Among the subjects enrolled, 8 were male and 2 were female, the age ranged from 30 to 58 years. Participants with T2D were grouped based on $\mathrm{HbA} 1 \mathrm{c}$ into three categories; $6.6-7.0 \%(\mathrm{n}=2), 7.1-8.5 \%(\mathrm{n}=2)$, and $>8.5 \%$ $(\mathrm{n}=1)$; the average BMI was 30.536 (Additional file 1: Table S1). A total of 2,431,348 and 2,972,433 raw sequence reads were generated from control subjects and T2D subjects, respectively. A total of 1,248,393 and $1,544,225$ rRNA sequences were identified in control subjects and T2D subjects, respectively. The average number of processed paired reads per sample was 292,773.6 reads for healthy controls and 359,210 reads for T2D subjects, respectively. The clustering of all the qualified sequences at the $97 \%$ similarity level generated a total of 8414 OTUs. From the healthy and T2D samples, 3887 (average 777.4 OTU per sample) and 4527 OTUs (average 905.4 OTU per sample) were detected, respectively (Additional file 1: Fig. S1; Table 1). The principal coordinate analysis plot of the unweighted UniFrac distance matrix distinguish DM2, DM3, and DM5 from the rest of the samples suggested the presence of different bacterial communities (Additional file 1: Fig. S2). At the phylum level, the common bacteria-Firmicutes, Bacteroidetes, Proteobacteria, and Actinobacteria were dominant among both T2D patients and healthy subjects. At the class level, most of the shared OTUs between T2D subjects and healthy subjects belonged to class Actinobacteria, Bacteroidia, Bacilli, Clostridia, Betaproteobacteria, and Gammaproteobacteria. The major bacterial families representing the gut microbiome profile of the subjects were Lachnospiraceae, Ruminococcaceae, Prevotellaceae, Bacteroidaceae, Enterobacteriaceae, and Bifidobacteriaceae. Prevotella copri, Bacteroides sp., Faecalibacterium prausnitzii, Butyricimonas sp., Bacteroides fragilis, Oscillospira

Table 1 Read count statistics of control and T2D patients

\begin{tabular}{llllr}
\hline Sample ID & $\begin{array}{l}\text { Total paired } \\
\text { end } \\
\text { reads }\end{array}$ & $\begin{array}{l}\text { Processed } \\
\text { reads }\end{array}$ & $\begin{array}{l}\text { Total } \\
\text { identified } \\
\text { rRNA } \\
\text { sequences }\end{array}$ & $\begin{array}{r}\text { Total } \\
\text { OTUs } \\
\text { Picked }\end{array}$ \\
\hline C1 & 359,322 & 215,480 & 183,565 & 659 \\
C2 & 528,285 & 314,104 & 264,448 & 1026 \\
C3 & 379,927 & 236,256 & 209,914 & 600 \\
C4 & 629,712 & 380,659 & 318,530 & 753 \\
C5 & 534,102 & 317,369 & 271,936 & 849 \\
DM1 & 524,791 & 312,518 & 269,809 & 754 \\
DM2 & 633,448 & 392,035 & 333,970 & 703 \\
DM3 & 360,215 & 224,639 & 202,970 & 894 \\
DM4 & 344,695 & 193,327 & 150,162 & 801 \\
DM5 & $1,109,284$ & 673,531 & 587,314 & 1375 \\
\hline
\end{tabular}


sp., Butyrivibrio sp., Dorea sp. and Dialister sp among T2D subjects and healthy subjects (Fig. 1). According to G test (http://qiime.org/scripts/group_significance.html), Prevotella copri, Lactobacillus ruminis, Lactobacillus vaginalis, Bifidobacterium adolescentis, Bifidobacterium longum, Bacteroides fragilis, Blautia producta, Dorea formicigenerans, Haemophilus parainfluenzae, Ruminococcus gnavus, and Bifidobacterium bifidum were found in

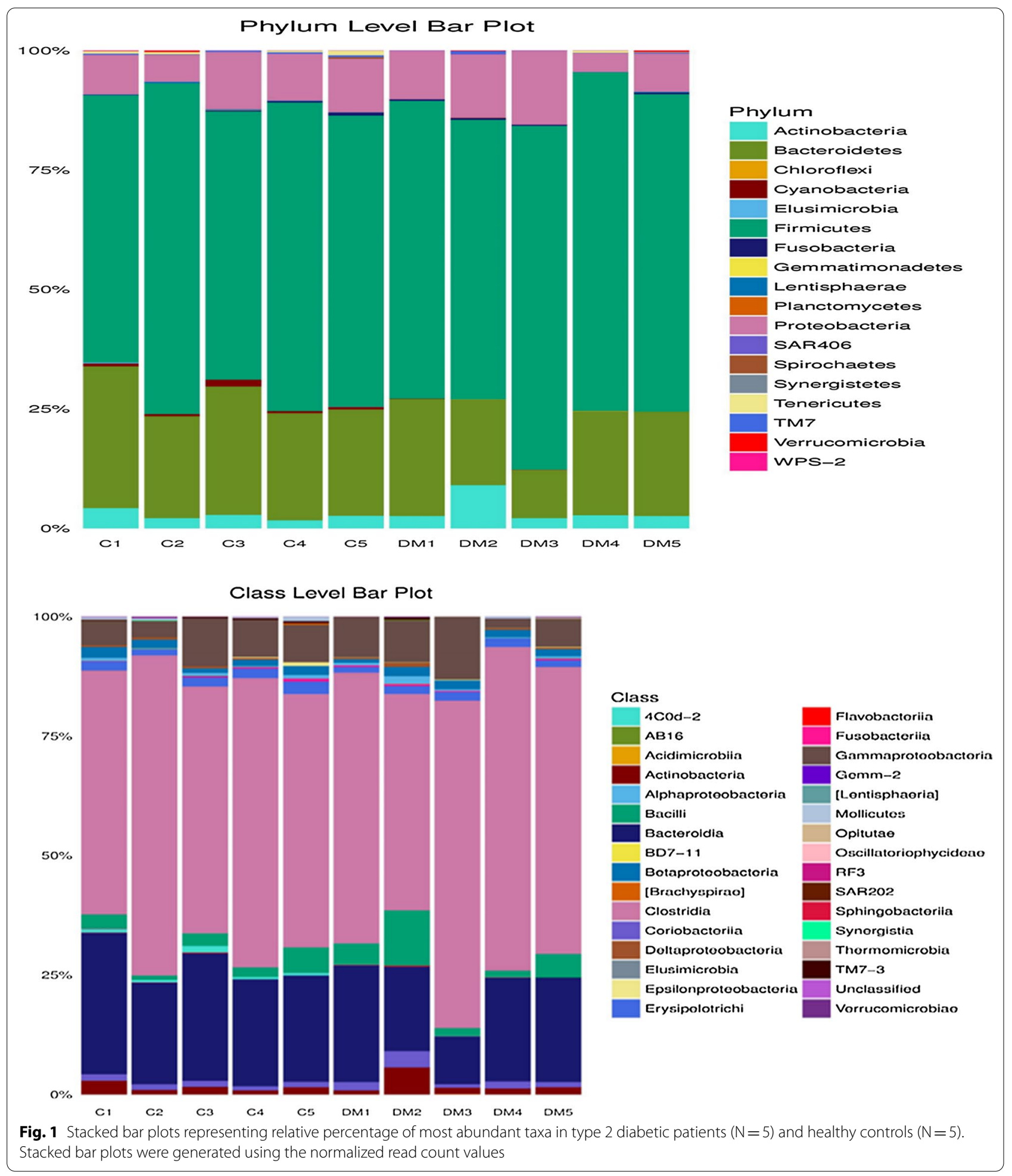




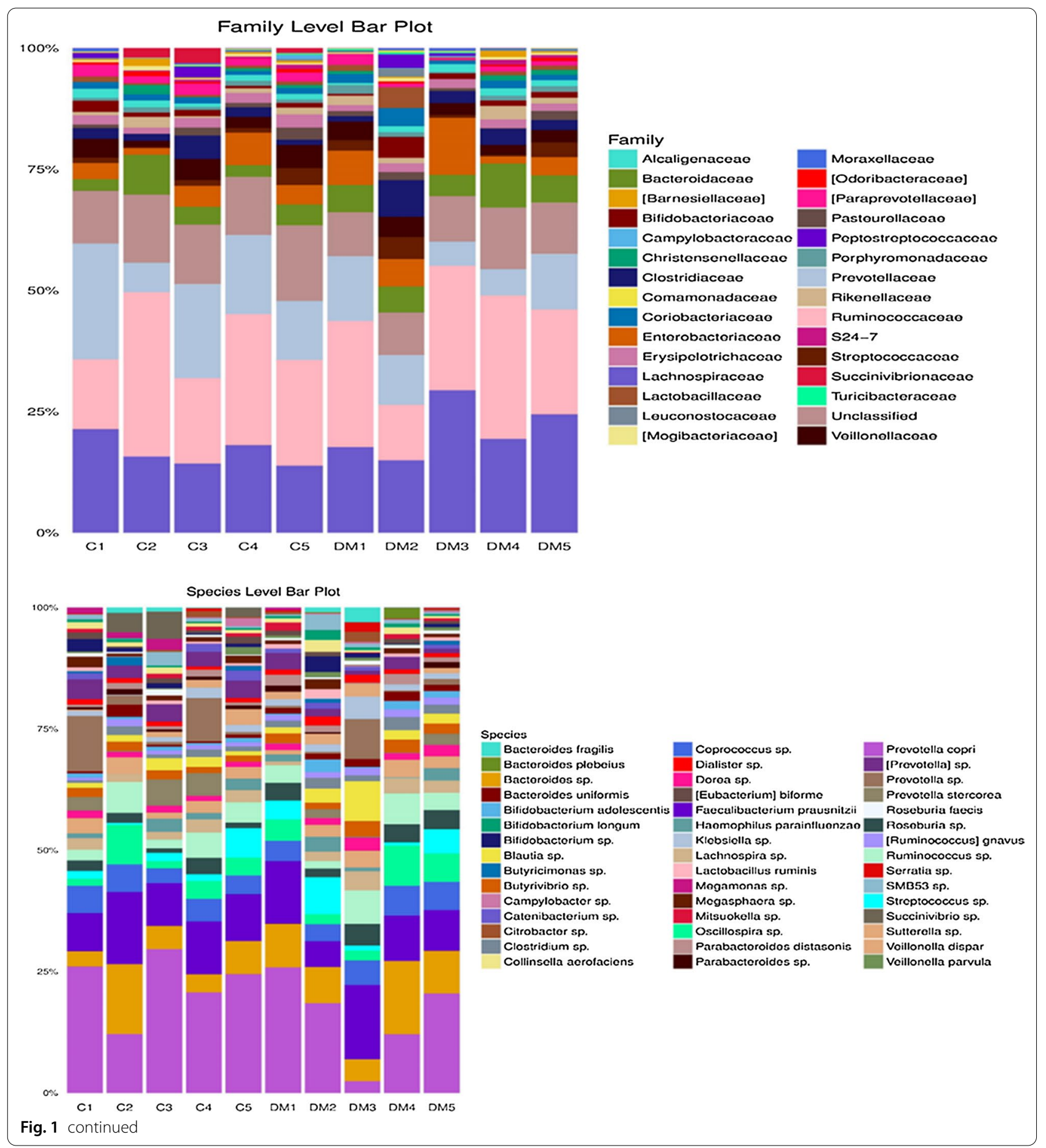

significantly higher abundance in T2D patients compared with healthy subjects. Of note, Bacteroides uniformis, Faecalibacterium prausnitzii, Prevotella stercorea, Veillonella parvula, Veillonella dispar, and Roseburia faecis had a significantly lower abundance in T2D patients compared with healthy subjects (Table 2).

\section{Discussion}

Evidence suggest that T2D development could be associated with a high levels of pro-inflammatory cytokines, chemokines and inflammatory proteins in the peripheral circulation. Given that lipopolysaccharides (LPS) of gram-negative bacteria induce TLR-4 complex-mediated 
Table 2 Comparison of Bacterial genus/species abundance in type II diabetes and healthy individuals with statistically significant difference $(P=0.05)$

\begin{tabular}{|c|c|c|c|c|}
\hline Bacterial Genus/species & $\begin{array}{l}\text { Mean abundance in Type } 2 \\
\text { Diabetes }\end{array}$ & $\begin{array}{l}\text { Mean abundance in Healthy } \\
\text { Individuals }\end{array}$ & $\begin{array}{l}\text { Status in Type } 2 \text { Diabetes } \\
\text { of our Study }\end{array}$ & Phylum \\
\hline Prevotella copri & 5123.6 & 3048 & Elevated & Bacteroidetes \\
\hline Lactobacillus ruminis & $14,550.4$ & 340.8 & Elevated & Firmicutes \\
\hline Lactobacillus vaginalis & 446.2 & 0.2 & Elevated & Firmicutes \\
\hline Bifidobacterium adolescentis & 73.8 & 3.6 & Elevated & Actinobacteria \\
\hline Bacteroides uniformis & 0.4 & 133.8 & Reduced & Bacteroidetes \\
\hline Faecalibacterium prausnitzii & 0.4 & 67.8 & Reduced & Firmicutes \\
\hline Bifidobacterium longum & 1718.8 & 228.2 & Elevated & Actinobacteria \\
\hline Ruminococcus & 954.6 & 102.6 & Elevated & Firmicutes \\
\hline Catenibacterium & 287.2 & 809 & Reduced & Firmicutes \\
\hline Coprococcus & 1695.6 & 841.2 & Elevated & Firmicutes \\
\hline Lachnospira & 984 & 221.8 & Elevated & Firmicutes \\
\hline Enterobacter cloacae & 251 & 31.8 & Elevated & Proteobacteria \\
\hline Butyrivibrio & 115.2 & 304.6 & Reduced & Firmicutes \\
\hline Klebsiella & 2255.4 & 537 & Elevated & Proteobacteria \\
\hline Haemophilus parainfluenzae & 592.4 & 17.8 & Elevated & Proteobacteria \\
\hline Mitsuokella multacida & 432.2 & 175 & Elevated & Firmicutes \\
\hline Prevotella stercorea & 0 & 409.8 & Reduced & Bacteroidetes \\
\hline Bacteroides fragilis & 11,082 & 716.6 & Elevated & Bacteroidetes \\
\hline Bifidobacterium bifidum & 98 & 0 & Elevated & Actinobacteria \\
\hline Veillonella parvula & 91.2 & 332.8 & Reduced & Firmicutes \\
\hline Hafnia alvei & 222.6 & 10.6 & Elevated & Proteobacteria \\
\hline Butyricimonas & 99 & 0 & Elevated & Bacteroidetes \\
\hline Bacteroides caccae & 296.6 & 10.2 & Elevated & Bacteroidetes \\
\hline Veillonella dispar & 221.2 & 478.8 & Reduced & Firmicutes \\
\hline Ruminococcus & 2.8 & 62.2 & Reduced & Firmicutes \\
\hline Megasphaera & 21,795 & 822 & Elevated & Firmicutes \\
\hline Collinsella aerofaciens & 492.4 & 326.8 & Elevated & Actinobacteria \\
\hline Roseburia faecis & 43.8 & 111.2 & Reduced & Firmicutes \\
\hline Dorea formicigenerans & 138.8 & 61.8 & Elevated & Firmicutes \\
\hline Blautia producta & 159.6 & 84.8 & Elevated & Firmicutes \\
\hline Citrobacter & 70.4 & 25.8 & Elevated & Proteobacteria \\
\hline Ruminococcus gnavus & 14.4 & 0.2 & Elevated & Firmicutes \\
\hline
\end{tabular}

inflammatory responses that may drive the potential development of T2D [9], we also aimed to determine the signatures of inflammation and anti-inflammation associated with the observed gut microbiome profiles in T2D. Lactobacillus spp., Bifidobacterium spp., Prevotella spp., Ruminococcus spp., and Bacteroides spp. generate acetate through the Wood-Ljungdahl and acetyl-CoA pathways. Veillonella spp., Bacteroides spp., Megasphaera sp, Roseburia sp., Ruminococcus sp., and Coprococcus sp are responsible for the production of propionate through succinate, acrylate, and propanediol pathways. Roseburia spp., Faecalibacterium prausnitzii, and Coprococcus sp are responsible for the production of butyrate through the butyryl-CoA:acetate CoA-transferase routes and the phosphotransbutyrylase/butyrate kinase pathway. Bacteroides fragilis, Lactobacillus, and Bifidobacterium are involved in the metabolism of secondary bile acids. SCFAs induce secretion of cytokines by intestinal epithelial cells (IECs) through MEK-ERK and p38 MAPK pathways via activation of G-protein coupled receptors (GPRs) 41 and 43 [10]. Binding of SCFAs to G-protein coupled receptors (GPCRs) drive the activation of RAS, PKA, PI3K, ERK1/2, and ATF2 signalling cascade, which induce the secretion and release of inflammatory mediators such as IL-1, MCP1, IL-6, TNF- $\alpha$, CXCL1, and CXCL2 [11]. This also activates the assembly of inflammasome and induces the secretion of Th1 polarising inflammatory cytokine, IL-18 [12] as well as IL-1 $\beta$, both 
via activation of caspase 1 . Firmicutes are gram-positive whereas Bacteroidetes are gram-negative bacteria. In obese individuals, a higher abundance of Firmicutes enhances the level of LPS that enhances the expression of inducible nitric oxide synthase (iNOS), which has been known to impair insulin sensitivity [13].

Faecalibacterium prausnitzii produces $15 \mathrm{kDa}$ protein that drives anti-inflammatory milieu through the inhibition of NF- $\mathrm{KB}$ pathway in intestinal epithelial cells [14]. Faecalibacterium prausnitzii is considered to be the main butyrate producing bacteria in the intestine and prevents inflammation through the production of metabolites that maintain the function of intestinal barrier. Butyrate inhibits inflammation of intestinal mucosa through activation of PPAR $\gamma$ and inhibition of NF-KB and IFN- $\gamma$ [15]. Butyrate induces hyperacetylation of histones through the prevention of histone deacetylase. F. prausnitzii augments anti-inflammatory activity through the expression of IL-10 in peripheral blood [16]. It is also involved in the fermentation of unabsorbed carbohydrate [17]. On the other hand, the lower abundance of $F$. prausnitzii reduces the level of butyrate production in the intestine. Butyrate induces the activation of free fatty acid receptor 2 that regulates the insulin signaling pathway in adipose tissue. It also induces the secretion of glucagon-like peptide 1 (GLP1) in the gut that prevents the accumulation of fat and enhances the sensitivity to insulin. Low level butyrate production due to lower abundance of $F$. prausnitzii induces the production of inflammatory cytokines through the activation of NF-kb [18].

Lactobacillus ruminis induces the secretion of proinflammatory cytokine IL- 8 by gut epithelial cells and is also involved in the elevation of IL- 6 in the serum of stroke patients [19]. An anaerobic gram-positive gut bacteria Ruminococcus gnavus is involved in the secretion of glucorhamnan polysaccharide with an L-rhamnose oligosaccharide backbone and glucose sidechains which drives the activation of TNF $\alpha$ in dendritic cells [20]. Butyrivibrio is a commensal bacteria involved in the production of butyrate through fermentation of pyruvate. Butyrate induces the secretion of an anti-inflammatory cytokine IL-10 from regulatory $\mathrm{T}$ cells (Treg). It also inhibits the proliferation of pathogenic bacteria in the gut by inducing secretion of IgA by plasma cells [21]. The abundance of Butyricimonas is associated with the production of inflammatory cytokines such as IL-1 $\beta$ and TGF $\beta 1$ in the ileum [22]. C. aerofaciens has genes that encode butyric acid kinase and phosphate butyryltransferase enzymes. It is involved in the biosynthesis of butyric acid which enhances inflammation in T2D [23]. OmpW protein produced by Bacteroides caccae induces an inflammatory response in clinical gout [24]. Bacteroides fragilis toxin (BFT) of $B$. fragilis induces activation of Stat 3 that drive antimicrobial peptide secretion and glycosylation of mucus. LpxF of $B$. fragilis allows to bypassing the LPS induced TLR4 dependent phagocytic activity [25]. Reduced abundance of $B$. uniformis strains has been associated with intestinal inflammation [26]. Higher abundance of Prevotella copri and IL-6 showed positive association with the develop of T2D [27].

In conclusion, our findings showed that a higher abundance of inflammatory bacteria such as Lactobacillus ruminis, Ruminococcus gnavus, Bacteroides caccae, Butyricimonas, Prevotella copri, and Collinsella aerofaciens and lower abundance of anti-inflammatory bacteria such as Faecalibacterium prausnitzii, and Butyrivibrio may have a role in the development of T2D. The current small sample size based pilot metagenomic investigation provides a snap short of diagnostic markers and treatment targets in T2D patients.

\section{Limitations}

This study was conducted with a limited number of T2D patients; thus, the gut bacterial signatures are required to validate in a larger cohort to understand the role of inflammatory and anti-inflammatory bacteria in T2D.

\section{Supplementary Information}

The online version contains supplementary material available at https://doi. org/10.1186/s13104-021-05466-2.

Additional file1: Figure $\mathrm{S} 1$. Rarefaction plot analysis of $\mathrm{V} 3$ sequencing of $16 \mathrm{~S}$ rRNA gene in faecal microbiota from T2D patients (DM1-DM5) and non-diabetic controls (C1-C5). Figure S2. Beta-diversity of the gut microbial communities in T2D patients and healthy controls. Principal Coordinates Analysis (PCOA) plot based on weighted (A) and unweighted (B) UniFrac distance. Each dot represents one sample from each group. Table S1. Clinical and demographic profile of type 2 diabetes mellitus subjects and healthy subjects.

Abbreviations

T2D: Type 2 diabetes; SCFAs: Short-chain fatty acids; OTU: Operational taxonomic unit.

\section{Acknowledgments}

The authors are grateful to Genotypic Technology Pvt Ltd, Bangalore, India for metagenomic sequencing services. The authors are grateful to Prof. E.M. Shankar, Department of Life Sciences, Central University of Tamil Nadu for critical inputs and insightful suggestions.

\section{Author contributions}

PK \& PD are involved in the clinical and diagnostic part of the study. IC analysed the data; wrote the manuscript and approved the final manuscript. All authors read and approved the final manuscript.

\section{Funding}

The authors are grateful to UGC-BSR Start-up grant of Govt. of India for financial support.

\section{Availability of data and materials}

The datasets utilized in present study are available in supplementary file and raw data of sequencing are available from the corresponding author on reasonable request. 


\section{Ethics approval and consent to participate}

All individuals included in the present study gave written informed consent before participation in the pilot study. The study protocol (TDU/IEC/AL/ 2017-18/10) was approved by the Ethical Committees of the Institute of Ayurveda and Integrative Medicine (I-AIM), Bangalore, India.

\section{Consent for publications}

Not applicable.

\section{Competing interests}

The authors declare no conflict of interest.

\section{Author details}

${ }^{1}$ Institute of Ayurveda and Integrative Medicine (I-AIM), Bangalore, India.

${ }^{2}$ Department of Life Sciences, Central University of Tamil Nadu, Thiruvarur 610005, India.

Received: 24 August 2020 Accepted: 28 January 2021

Published online: 06 February 2021

\section{References}

1. Roberts LD, Koulman A, Griffin JL. Towards metabolic biomarkers of insulin resistance and type 2 diabetes: progress from the metabolome. Lancet Diabetes Endocrinol. 2014:2:65-75.

2. Baothman OA, Zamzami MA, Taher I, Abubaker J, Abu-Farha M. The role of Gut Microbiota in the development of obesity and Diabetes. Lipids Health Dis. 2016:15:108.

3. Tai N, Wong FS, Wen L. The role of gut microbiota in the development of type 1, type 2 diabetes mellitus and obesity. Rev Endocr Metab Disord. 2015;16(1):55-65.

4. Ma Q, Li Y, Li P, Wang M, Wang J, Tang Z, Wang T, Luo L, Wang C, Wang T, Zhao B. Research progress in the relationship between type 2 diabetes mellitus and intestinal flora. Biomed Pharmacother. 2019:117:109138.

5. Karlsson FH, Tremaroli V, Nookaew I, et al. Gut metagenome in European women with normal, impaired and diabetic glucose control. Nature. 2013:498:99-103.

6. Tilg H, Moschen AR. Microbiota and diabetes: an evolving relationship. Gut. 2014:63(9):1513-21.

7. Li Q, Chang Y, Zhang K, Chen H, Tao S, Zhang Z. Implication of the gut microbiome composition of type 2 diabetic patients from northern China. Sci Rep. 2020;10(1):5450.

8. Ahmad A, Yang W, Chen G, Shafiq M, Javed S, Ali Zaidi SS, Shahid R, Liu $\mathrm{C}$, Bokhari $\mathrm{H}$. Analysis of gut microbiota of obese individuals with type 2 diabetes and healthy individuals. PLoS ONE. 2019;14(12):e0226372.

9. Larsen N, Vogensen FK, van den Berg FW, Nielsen DS, Andreasen AS, Pedersen BK, Al-Soud WA, Sørensen SJ, Hansen LH, Jakobsen M Gut microbiota in human adults with type 2 diabetes differs from non-diabetic adults. PLoS ONE. 2010;5(2):e9085.

10. Sittipo P, Shim JW, Lee YK. Microbial metabolites determine host health and the status of some diseases. Int J Mol Sci. 2019;20(21):5296.

11. Kim CH, Park J, Kim M. Gut microbiota-derived short-chain Fatty acids, $T$ cells, and inflammation. Immune Netw. 2014;14(6):277-88.

12. Rooks MG, Garrett WS. Gut microbiota, metabolites and host immunity. Nat Rev Immunol. 2016;16(6):341-52.

13. Saad MJ, Santos A, Prada PO. Linking gut microbiota and inflammation to obesity and insulin resistance. Physiology (Bethesda). 2016;31(4):283-93.
14. Bajer L, Kverka M, Kostovcik M, Macinga P, Dvorak J, Stehlikova Z, Brezina J, Wohl P, Spicak J, Drastich P. Distinct gut microbiota profiles in patients with primary sclerosing cholangitis and ulcerative colitis. World J Gastroenterol. 2017;23(25):4548-58.

15. Lopez-Siles M, Duncan SH, Garcia-Gil LJ, Martinez-Medina M. Faecalibacterium prausnitzii: from microbiology to diagnostics and prognostics. ISME J. 2017:11(4):841-52.

16. Rabiei N, Ahmadi Badi S, Ettehad Marvasti F, Nejad Sattari T, Vaziri F, Siadat SD. Induction effects of Faecalibacterium prausnitzii and its extracellular vesicles on toll-like receptor signaling pathway gene expression and cytokine level in human intestinal epithelial cells. Cytokine 2019:121:154718.

17. Del Chierico F, Abbatini F, Russo A, Quagliariello A, Reddel S, Capoccia D, Caccamo R, Ginanni Corradini S, Nobili V, De Peppo F, Dallapiccola B, Leonetti F, Silecchia G, Putignani L. Gut microbiota markers in obese adolescent and adult patients: age-dependent differential patterns. Front Microbiol. 2018;9:1210.

18. Fassatoui M, Lopez-Siles M, Díaz-Rizzolo DA, Jmel H, Naouali C, Abdessalem G, Chikhaoui A, Nadal B, Jamoussi H, Abid A, Gomis R, Abdelhak S, Martinez-Medina M, Kefi R. Gut microbiota imbalances in Tunisian participants with type 1 and type 2 diabetes mellitus. Biosci Rep. 2019. https://doi.org/10.1042/BSR20182348.

19. Yamashiro K, Tanaka R, Urabe T, Ueno Y, Yamashiro Y, Nomoto K, Takahashi T, Tsuji H, Asahara T, Hattori N. Gut dysbiosis is associated with metabolism and systemic inflammation in patients with ischemic stroke. PLoS ONE. 2017;12(2):e0171521.

20. Henke MT, Kenny DJ, Cassilly CD, Vlamakis H, Xavier RJ, Clardy J. Ruminococcus gnavus, a member of the human gut microbiome associated with Crohn's disease, produces an inflammatory polysaccharide. Proc Nat Acad Sci USA. 2019;116:12672-7.

21. Anand S, Kaur H, Mande SS. Comparative in silico analysis of butyrate production pathways in gut commensals and pathogens. Front Microbiol. 2016;7:1945.

22. Kim J, Lee H, An J, Song Y, Lee CK, Kim K, Kong H. Alterations in gut microbiota by statin therapy and possible intermediate effects on hyperglycemia and hyperlipidemia. Front Microbiol. 2019;10:1947.

23. Qin P, Zou Y, Dai Y, Luo G, Zhang X, Xiao L. Characterization a novel butyric acid-producing bacterium Collinsella aerofaciens subsp. shenzhenensis subsp. nov. Microorganisms. 2019;7(3):78.

24. Guo Z, Zhang J, Wang Z, Ang KY, Huang S, Hou Q, Su X, Qiao J, Zheng Y, Wang L, Koh E, Danliang H, Xu J, Lee YK, Zhang H. Intestinal microbiota distinguish gout patients from healthy humans. Sci Rep. 2016:6:20602.

25. Casterline BW, Hecht AL, Choi VM, Bubeck WJ. The Bacteroides fragilis pathogenicity island links virulence and strain competition. Gut Microbes. 2017:8(4):374-83.

26. Bruzzese E, Callegari ML, Raia V, et al. Disrupted intestinal microbiota and intestinal inflammation in children with cystic fibrosis and its restoration with Lactobacillus GG: a randomised clinical trial. PLOS ONE. 2014;9(2):e87796.

27. Leite AZ, Rodrigues NC, Gonzaga MI, et al. Detection of increased plasma interleukin-6 levels and prevalence of prevotella copri and Bacteroides vulgatus in the feces of type 2 diabetes patients. Front Immunol. 2017:8:1107.

\section{Publisher's Note}

Springer Nature remains neutral with regard to jurisdictional claims in published maps and institutional affiliations. 